\title{
Verapamil COER-24 180/240mg na Hipertensão Arterial Leve a Moderada em Dose Única Diária Avaliado pela Monitorização Ambulatorial da Pressão Arterial
}

\author{
Katia Coelho Ortega, José Luis Santello, Fernando Nobre, Oswaldo Kohlman Jr, Paulo César B. Veiga \\ Jardim, Lilian Soares da Costa, Guido Aranha Rosito, José Joaquim Fernandes Raposo F, \\ Wille Oigman, Décio Mion Jr
}

São Paulo, SP - Brazil

\begin{abstract}
Objetivo - Avaliar a eficácia terapêutica do verapamil COER-24 180/240 mg, em dose única, ao deitar, como monoterapia para a hipertensão arterial leve a moderada.
\end{abstract}

Métodos - Estudo multicêntrico, aberto, não comparativo com 81 pacientes de ambos os sexos, com idade $>20$ anos e hipertensão arterial essencial leve e moderada. Medimos a pressão arterial no consultório e com a monitorização ambulatorial (MAPA) durante 24h antes e ao final de 8 semanas do uso da medicação.

Resultados - Verificou-se diminuição $(p<0,0001)$ das pressões sistólicas e diastólicas medidas no consultório às semanas 3 e 8. A MAPA demonstrou que tanto a pressão sistólica, diastólica, média e freqüência cardíaca, quando as cargas pressóricas médias de 24 h apresentaram reduções após 8 semanas de tratamento, além da redução do duplo-produto, especialmente pela manhã. A tolerabilidade foi boa, $68 \%$ dos pacientes não apresentaram eventos adversos.

Conclusão - A monoterapia com o verapamil COER24 180/240mg em dose única é eficaz para o controle da pressão arterial em hipertensos leves e moderados, com redução tanto na pressão casual quanto na MAPA/24h, além de apresentar boa tolerabilidade.

Palavras-chave: verapamil, hipertensão arterial, MAPA

\section{Single Daily Dose of Verapamil (COER-24 $180 / 240 \mathrm{mg}$ ) in Mild and Moderate
Hypertension Evaluated by Ambulatory Blood Pressure Monitoring

Objective - To evaluate the anti-hypertensive effecft of verapamil COER-24 180/240 mg in a single dose at bedtime as single therapy in mild to moderate hypertensives.

Methods - A multicentric, open, placebo controlled study of 81 hypertensive patients older than 20 years-old followed to 8 weeks. Blood pressure was measured in doctor's office and by 24 h ambulatory monitoring (ABPM).

Results - We observed a decreased in systolic and diastolic blood pressure in doctor's office at $4^{\text {th }}$ and $8^{\text {th }}$ weeks. ABPM showed that both systolic, diastolic and mean blood pressure, heart rate and the mean 24-hour blood pressure load decreased after the 8-week treatment. In addition, there was a reduction of the double-product, especially in the morning and $68 \%$ of the patients didn't have any adverse events.

Conclusion - The theraphy verapamil COER-24 180/ $240 \mathrm{mg}$ in a single dose is useful for mild and moderate hypertensive patients, with significant pressure decrease in both office blood pressure measurements and in the ABPM/24 hours, as well as showing good tolerability.

Keywords: verapamil, hypertension, ABPM.

Arq Bras Cardiol, volume 74 (n' 3), 283-290, 2000

Grupo de Estudos de Cronovera - Brasil

Correspondência: Décio Mion Jr. - Unidade de Hipertensão do Hospital das Clínicas

- Av. Dr. Enéas C. Aguiar, 255 - $7^{\circ}$ - S/7032 - 05403-000 - São Paulo, SP

Recebido para publicação em 21/10/99

Aceito em 15/3/00
A hipertensão arterial é importante fator de risco para complicações cardiovasculares que, de acordo com evidências recentes, apresentam distribuição circadiana. Assim, eventos cardiovasculares, como infarto agudo do miocárdio, morte súbita e acidente vascular cerebral apresen- 
tam pico de incidência durante o período da manhã, provavelmente devido ao tônus simpático e à coagulabilidade sangüínea aumentados nestes períodos. Deste modo, torna-se lógico que o tratamento anti-hipertensivo deva objetivar, principalmente, este momento mais crítico ${ }^{1-3}$.

Por outro lado, a pressão arterial, na maioria dos pacientes hipertensos, também apresenta padrão circadiano caracterizado por aumento abrupto pela manhã após o despertar, níveis mais elevados durante toda a atividade diurna e atenuação da redução da pressão durante o sono ${ }^{4}$. Do ponto de vista de risco cardiovascular, a ausência de descenso da pressão arterial durante o sono tem sido considerado fator de risco de lesão aos órgãos-alvo, doença cardíaca isquêmica e acidente vascular cerebral ${ }^{5-9}$. Assim, frente a estes dados também parece-nos lógico que o tratamento anti-hipertensivo deve controlar sem ocasionar quedas excessivas da pressão durante o sono, atuando durante o período de vigília quando ela está mais elevada, sobretudo, no período da manhã.

A cronoterapia é uma modalidade de tratamento que procura parear os efeitos do tratamento com os sintomas da doença pela alteração proposital da concentração da droga durante as $24 \mathrm{~h}$, levando em consideração a sincronia com os ritmos circadianos conhecidos na atividade da doença e no risco de eventos clínicos, por um lado, e os efeitos desejados e adversos dos medicamentos, por outro lado, a fim de otimizar os resultados terapêuticos.

A primeira cronoterapia disponível para hipertensão arterial foi introduzida nos Estados Unidos em 1996 e no Brasil, Canadáe México em 1997. O verapamil de ação programadae liberação prolongada, denominado verapamil COER-24 da abreviatura inglesa "COER-24" correspondente a controlled extended release for 24 hours, utiliza uma tecnologia sofisticada de liberação da droga para parear a concentração do medicamento durante as $24 \mathrm{~h}$ do dia, em sincronia com o padrão vigília-sono da pressão arterial ${ }^{10}$.

Baseados nestas informações, realizou-se estudo multicêntrico, aberto, controlado com placebo, objetivando avaliar a eficácia terapêutica do verapamil COER-24 180/240mg, em dose única, como monoterapia para a hipertensão arterial leve a moderada e a resposta anti-hipertensiva, através das medidas casuais e da monitorização ambulatorial da pressão arterial durante $24 \mathrm{~h}$, obtidas antes e ao final do uso da medicação.

\section{Métodos}

Foram selecionados pacientes de ambos os sexos, com idade > 20 anos, e diagnóstico de hipertensão arterial essencial definida por diastólica entre 90 e $114 \mathrm{mmHg}$ em medidas casuais, após consentimento por escrito para participação. O estudo foi aprovado pelas Comissões de Ética das Instituições participantes, a saber: Décio Mion Jr, Katia Coelho Ortega e José Luis Santello, Hospital das Clínicas da Faculdade de Medicina da Universidade de São Paulo - 25 pacientes; Fernando Nobre, Hospital das Clínicas de Ribeirão Preto da Faculdade de Medicina de Ribeirão Preto da Universidade de São Paulo - 7 pacientes; Guido Bernardo Rosito, Hospital de Clínicas de Porto Alegre - 10 pacientes; José
Joaquim Fernandes Rapozo $F^{\mathbf{o}}$, Ecocenter - Centro de Cardiologia não Invasiva - 5 pacientes; Lilian Soares Da Costa, Hospital Santa Casa da Misericórdia do Rio de Janeiro - 10 pacientes; Osvaldo Kohlman, Escola Paulista de Medicina da Universidade Federal de São Paulo - 12 pacientes; Paulo César B. Veiga Jardim, Hospital das Clínicas da Faculdade de Medicina da Universidade Federal de Goiás - 15 pacientes; Wille Oigman, Hospital Universitário Pedro Ernesto da Universidade Estadual do Rio de Janeiro - 15 pacientes. $O$ período do estudo foi de setembro/97 a janeiro/99.

Foram excluídos do estudo pacientes que apresentassem as seguintes condições: infarto do miocárdio ou acidente vascular cerebral há menos de seis meses da data de inclusão no estudo, bloqueio atrioventricular de $2^{\circ}$ ou $3^{\circ}$ graus, insuficiência cardíaca graus III ou IV de acordo com os critérios da American Heart Association, hipertensão secundária, bradicardia com freqüência cardíaca abaixo de $60 \mathrm{bpm}$, insuficiência renal ou hepática, presença de gravidez ou pacientes do sexo feminino potencialmente capazes de engravidar, pacientes que demonstraram falta de cooperação ou que não manifestaram interesse em participar do estudo e portadores de outras doenças graves de qualquer natureza.

Os pacientes foram submetidos a anamnese, exame físico e medida da pressão arterial. Aqueles que preencheram os critérios de inclusão tiveram suas medicações anti-hipertensivas prévias suspensas e receberam placebo durante o período de 14 dias. Após esse período, caso a diastólica estivesse entre 90 e $114 \mathrm{mmHg}$ em medidas casuais, os pacientes iniciaram a fase de tratamento ativo após o registro de monitorização ambulatorial da pressão arterial durante $24 \mathrm{~h}$.

Durante as quatro primeiras semanas do tratamento, os pacientes receberam verapamil COER-24 180mg, um comprimido, via oral, ao deitar. Após esse período, os pacientes foram submetidos a nova avaliação incluindo exame físico, medida da pressão e avaliação dos efeitos colaterais. Nessa ocasião, caso a diastólica estivesse $\geq 90 \mathrm{mmHg}$, a dose de verapamil COER-24 era aumentada para $240 \mathrm{mg}$ em dose única ao deitar, sendo mantida por mais quatro semanas. Caso contrário, se a diastólica estivesse $<90 \mathrm{mmHg}$ os pacientes continuavam recebendo verapamil COER-24 180mg durante as quatro semanas seguintes. Ao final desse período os pacientes foram submetidos a novo exame clínico, medida da pressão casual, avaliação de efeitos colaterais e a nova monitorização ambulatorial da pressão arterial de $24 \mathrm{~h}$.

A pressão arterial foi avaliada após $10 \mathrm{~min}$ de repouso na posição supina, por duas vezes consecutivas, com intervalo de 2 min entre cada uma delas, utilizando-se esfigmomanômetro de coluna de mercúrio calibrado. Terminadas as avaliações nesta posição, os pacientes permaneceram em posição ortostática por mais 3 min e foi realizada nova medida de pressão arterial para análise de possível hipotensão ortostática. Para efeito de referência foi considerada a média das duas medidas na posição supina.

A monitorização ambulatorial da pressão arterial de $24 \mathrm{~h}$ foi realizada utilizando-se o equipamento SpaceLabs 90207, validado pela Associação para o Avanço de Equipamentos Médicos (AAMI) e pela Sociedade Britânica 
de Hipertensão (BHS), em dois momentos distintos do estudo: após a fase placebo e após o período de 8 semanas de tratamento ativo, com medidas a cada $15 \mathrm{~min}$ no período de 6:00 às 23:00h e a cada 20min das 23:01 às 5:59h, empregando-se critérios de exclusão automática. O manguito foi colocado em braço não dominante, num dia representativo da atividade do paciente, após orientação sobre preenchimento de diário e demais cuidados para o bom sucesso do exame.

Para avaliação de eficácia, foi considerado sucesso terapêutico o paciente que apresentasse, ao final do período de tratamento ativo de oito semanas, níveis de diastólica inferiores a $90 \mathrm{mmHg}$ e como resposta terapêutica a redução de pelo menos $10 \mathrm{mmHg}$, na diastólica, em medidas casuais.

A resposta hipotensora foi avaliada através da comparação dos dados obtidos nas avaliações das pressões casuais antes, após quatro semanas e após oito semanas do tratamento com verapamil COER-24 180/240mg e das médias de pressão da monitorização ambulatorial da pressão arterial antes e após oito semanas do tratamento ativo, utilizandose análise de variância (ANOVA), considerando-se como estatísticamente significativo $\mathrm{p}<0,05$.

A tolerabilidade ao tratamento foi avaliada de forma subjetiva pelo pesquisador como: a) boa - ausência de efeitos adversos; b) regular - presença de efeito adverso sem necessidade de interrupção da medicação; e c) ruim - presença de efeito adverso havendo necessidade de interrupção da medicação. Os pacientes, ao final do estudo, avaliaram subjetivamente a qualidade de vida com o tratamento, como sendo ótima, boa, regular ou ruim.

\section{Resultados}

Foram selecionados 99 pacientes sendo que 18 foram excluídos devido a: elevação da pressão arterial na fase placebo incompatível com a manutenção no estudo (1), resposta ao placebo com normalização da pressão arterial (4), aban-

\begin{tabular}{|lc|}
\hline \multicolumn{2}{|c|}{ Tabela I - Características dos pacientes $(\mathbf{n = 8 1})$} \\
\hline Idade (anos) & $53,2 \pm 9,8$ \\
Sexo n (\%) & \\
Feminino & $56(69)$ \\
Masculino & $25(31)$ \\
Raça n (\%) & \\
Branca & $47(58)$ \\
Negra & $21(26)$ \\
Parda & $7(9)$ \\
Amarela & $2(2)$ \\
Sem Informação & $4(5)$ \\
Peso (kg) & $69,7 \pm 11,4$ \\
Altura (m) & $1,6 \pm 0,1$ \\
Índice de massa corporal $\left(\mathrm{kg} / \mathrm{m}^{2}\right)$ & $27,4 \pm 3,9$ \\
Pulso (bpm) & $72,9 \pm 9,1$ \\
Pressão sistólica supina $(\mathrm{mmHg})$ & $151,3 \pm 15,4$ \\
Pressão diastólica supina $(\mathrm{mmHg})$ & $97,7 \pm 6,6$ \\
Pressão sistólica ortostática $(\mathrm{mmHg})$ & $149,6 \pm 16,0$ \\
Pressão diastólica ortostática $(\mathrm{mmHg})$ & $98,2 \pm 6,9$ \\
\hline Média \pm DP. & \\
\hline
\end{tabular}

dono do estudo (6), solicitação do paciente para ser retirado do estudo devido a eventos adversos (5) e retirados do estudo a critério do investigador (2), restando para a análise final 81 pacientes. Os eventos adversos que motivaram a descontinuação do estudo em 5\% dos pacientes foram: palpitações e mal-estar (1), broncoespasmo (1), noctúria (1), intolerância gastrintestinal caracterizada por vômitos de difícil tratamento (1) e reação alérgica (1). As características dos 81 pacientes incluídos na visita inicial do estudo encontramse na tabela I.

Os pacientes apresentavam os seguintes antecedentes patológicos: angioplastia coronariana (1), dislipidemia (3), úlcera duodenal (1), gastrite (1), asma (2), doença pulmonar obstrutiva crônica (2), prostatismo (1), infecção urinária (1), litíase renal (1), síndrome nefrótica (1), gota (1), artrose (1), artrite reumatóide (1), osteoporose (2), diabetes mellitus (4), varizes em membros inferiores (1), hérnia inguinal (1)e labirintite (1).

Em 37 (49,33\%) dos pacientes a dose de varapamil COER-24 foi mantida em 180mg porque a diastólica estava abaixo de $90 \mathrm{mmHg}$ após quatro semanas de tratamento. Por outro lado, a diastólica permaneceu acima ou igual a $90 \mathrm{~m}$ $\mathrm{mHg}$ em $38(50,67 \%)$ pacientes que tiveram a dose de verapamil COER-24 aumentada para $240 \mathrm{mg}$ após quatro semanas do tratamento ativo.

Durante o estudo não ocorreu variação do peso e do índice de massa corpórea quando foram comparadas as semanas após o período placebo (semana 0 ), após quatro semanas do tratamento ativo (semana 4 ) e após oito semanas de tratamento ativo (semana 8).

Houve diminuição estatísticamente significante do pulso quando foram comparadas a visita após o período placebo à visita após oito semanas do tratamento ativo ( $\mathrm{F}=$ $5,71 ; \mathrm{P}=0,005)$.

Medida casual da pressão arterial - Verificou-se diminuição estatisticamente significante $(\mathrm{P}<0,0001)$ da sistólica e da diastólica medidas no consultório tanto na posição supina quanto ortostática, quando foram comparadas a semana 0 às semanas $4 \mathrm{e} 8$ (tabela II).

Após oito semanas do tratamento ativo, 39 (48\%) pacientes apresentavam diastólica na posição supina abaixo de $90 \mathrm{mmHg}$, enquanto $47(58 \%)$ pacientes apresentavam diastólica abaixo de $90 \mathrm{mmHg}$ na posição ortostática.

Após quatro semanas de tratamento ativo, na posição supina, $53(65,4 \%)$ pacientes apresentaram redução acima de $10 \mathrm{mmHg}$ da diastólica em relação à semana 0 , quando foi iniciado o tratamento ativo, enquanto após oito semanas do tratamento ativo, $63(77,8 \%)$ pacientes apresentaram redução acima de $10 \mathrm{mmHg}$ da diastólica em relação às medidas feitas após quatro semanas do tratamento. Na posição ortostática, $58(71,6 \%)$ pacientes apresentaram redução $>10 \mathrm{mmHg}$ quando foram comparadas as medidas feitas na semana 0 e após quatro semanas do tratamento ativo, assim como quando foram comparadas as medidas feitas após quatro e oito semanas do tratamento ativo.

Monitorização ambulatorial da pressão arterial - A análise da monitorização ambulatorial da pressão arterial 


\begin{tabular}{|c|c|c|c|c|c|}
\hline & Semana o & Semana 4 & Semana 8 & $\mathrm{P}$ & $\mathrm{F}$ \\
\hline Peso & $69,7 \pm 11,3$ & $69,4 \pm 11,3$ & $69,7 \pm 11,5$ & 0,024 & 3,9 \\
\hline $\mathrm{IMC}\left(\mathrm{kg} / \mathrm{m}^{2}\right)$ & $27,4 \pm 3,9$ & $27,3 \pm 3,9$ & $27,4 \pm 3,9$ & 0,016 & 4,3 \\
\hline Pulso (bpm) & $72,9 \pm 8,6$ & $70,9 \pm 9,5$ & $69,8 \pm 8,8$ & $0,005^{*}$ & 5,7 \\
\hline PAS (mmHg) & $156,7 \pm 14,7$ & $146,0 \pm 14,8$ & $143,9 \pm 14,6$ & $<0,0001 * *$ & 25,3 \\
\hline PAD (mmHg) & $100,2 \pm 6,6$ & $93,4 \pm 9,8$ & $91,4 \pm 9,8$ & $<0,0001 * *$ & 41,5 \\
\hline \multicolumn{6}{|l|}{ PA ortostática } \\
\hline PAS (mmHg) & $155,3 \pm 14,9$ & $143,8 \pm 15,9$ & $141,7 \pm 14,9$ & $<0,0001 * *$ & 31,5 \\
\hline PAD (mmHg) & $101,0 \pm 7,1$ & $94,4 \pm 10,7$ & $91,7 \pm 11,0$ & $<0,0001^{*} *$ & 44,5 \\
\hline
\end{tabular}

pré e pós tratamento mostra que tanto a pressão sistólica, diastólica, pressão média, freqüência cardíaca quanto as cargas pressóricas médias de $24 \mathrm{~h}$ apresentaram reduções estatisticamente significantes após oito semanas de tratamento (fig. 1). Considerando-se o período de vigília (7:00 às 23:00h), verificou-se que as pressões arteriais sistólicas, diastólicas, médias e as cargas pressóricas médias também apresentaram reduções estatistica-

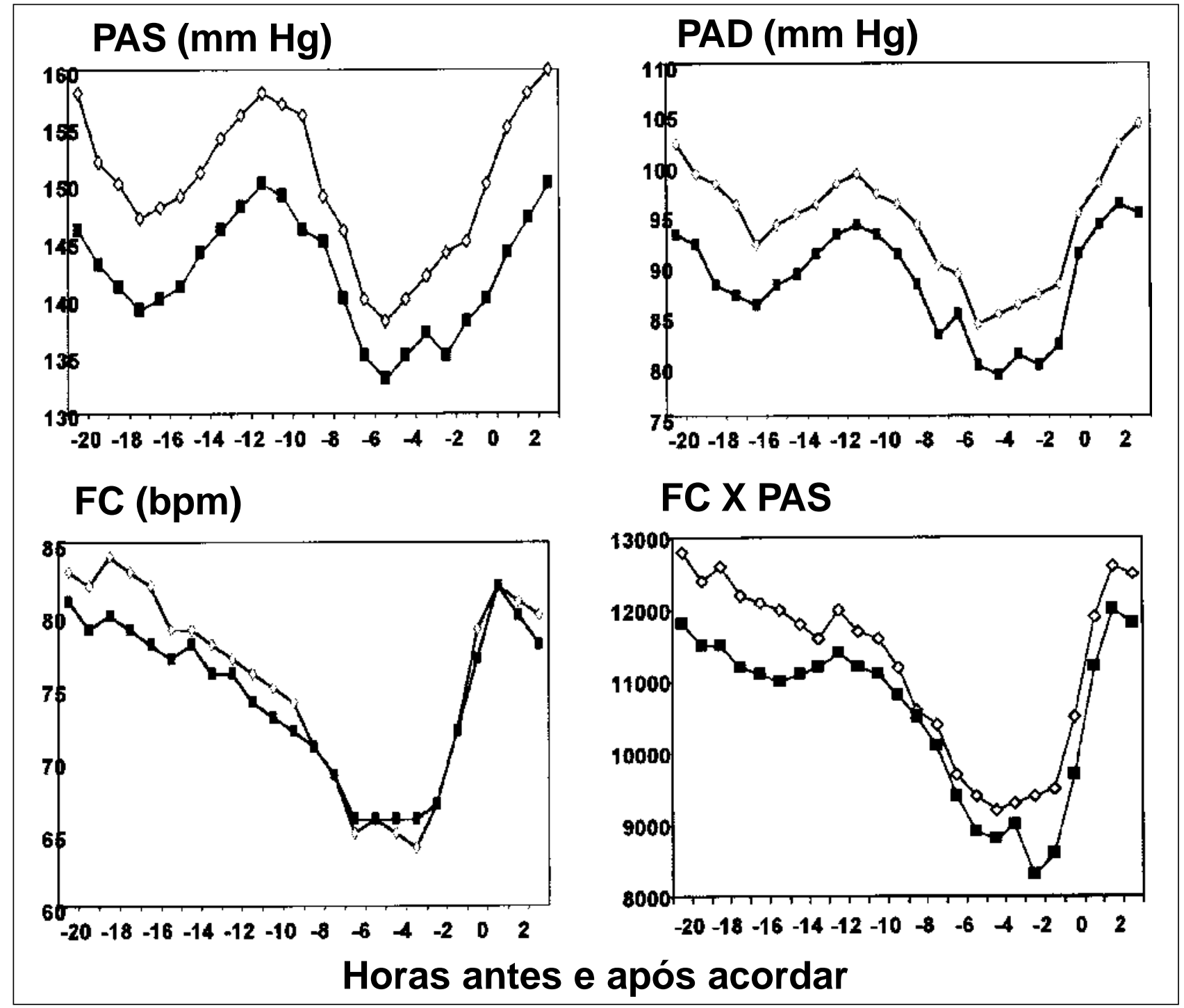

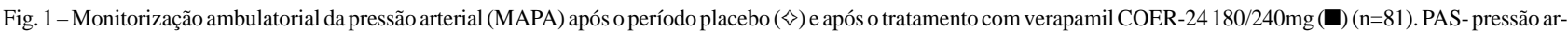
terial sistólica; PAD- pressão arterial diastólica; FC- freqüência cardíaca. 
mente significantes na segunda monitorização ambulatorial da pressão arterial. Durante o período de sono (23:01 às 6:59h), também ocorreram reduções estatisticamente significantes das pressões sistólicas, diastólicas, médias e cargas pressóricas na segunda monitorização ambulatorial da pressão arterial.

Com relação à freqüência cardíaca não se verificou variação antes e após o tratamento tanto para o período de vigília quanto para o de sono (tab. III).

O tratamento com verapamil COER-24 também reduziu o duplo-produto (frequiência cardíaca vs sistólica), uma medida indireta correspondente à demanda de oxigênio do miocárdio, especialmente pela manhã, quando a isquemia é de grande risco, conforme demonstrado na figura 1.

A análise das duas monitorização ambulatorial da pressão arterial, em períodos de $3 \mathrm{~h}$, mostrou que a maior diferença percentual da sistólica entre a primeira e a segunda monitorização ambulatorial da pressão arterial ocorreu no período das 6:00h às 9:00h $(-6,82 \pm 8,28)$ e a menor diferença percentual no período das $23: 00 \mathrm{~h}$ às $2: 00 \mathrm{~h}(-3,57 \pm 8,87)$, o mesmo ocorrendo para a diastólica, com a maior diferença entre a primeira e a segunda monitorização ambulatorial da

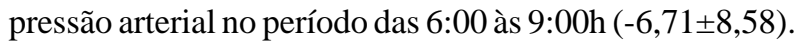
Porém, a menor diferença percentual entre a diastólica da primeira e da segunda monitorização ambulatorial da pressão arterial ocorreu no período das 18:00 às 22:00h ($3,25 \pm 9,14)$. A maior diferença percentual da pressão arterial média entre a primeira e a segunda monitorização ambulato-
Verapamil COER-24 180/240mg na HA avaliado pela MAPA

rial da pressão arterial ocorreu no período das 6:00 às 9:00h $(-7,21 \pm 8,25)$ e a menor diferença percentual no período das

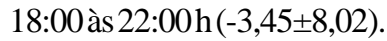

A avaliação das médias horárias da sistólica, diastólica, pressão arterial média, frequiência cardíaca e do duplo produto (freqüência cardíaca vs sistólica) nos exames de monitorização ambulatorial da pressão arterial realizados antes e após o tratamento ativo, no período de $3 \mathrm{~h}$ antes e após os pacientes acordarem, considerando-se o horário de acordar relatado pelos pacientes em seus respectivos diários, mostrou que ocorreu redução estatisticamente significante das médias da sistólica, diastólica, pressão arterial média e do duplo produto entre a primeira e a segunda monitorização ambulatorial DA pressão arterial, porém o mesmo não foi verificado com a frequiência cardíaca (fig. 2, tabs. IV e V).

Tolerabilidade - Os pacientes apresentaram os seguintes eventos adversos durante o decorrer do estudo: cefaléia (9), tontura (1), epigastralgia (3), edema de membros inferiores (1), broncoespasmo (2), diminuição da libido (1), dor precordial (2), visão embaçada (1), constipação (1).

A tolerabilidade ao medicamento, na opinião dos pesquisadores foi considerada boa em 61 (75\%) pacientes e regular em 17 (21\%) pacientes, não havendo informações de $3(4 \%)$ pacientes. A avaliação subjetiva do paciente referente à qualidade de vida, ao final do estudo foi considerada ótima para $34(42 \%)$, boa para $37(46 \%)$, regular para 6 (7\%) e ruim para $1(1 \%)$, não havendo informação de $3(4 \%)$ pacientes.

\begin{tabular}{|c|c|c|c|c|}
\hline & MAPA 1 & MAPA 2 & $P$ & $\mathrm{t}$ \\
\hline \multicolumn{5}{|l|}{ Médias durante $24 \mathrm{~h}$} \\
\hline Sistólica (mmHg) & $149,4 \pm 17,5$ & $140,9 \pm 15,7$ & $<0,0001$ & 7,9 \\
\hline Diastólica $(\mathrm{mmHg})$ & $94,0 \pm 11,6$ & $88,2 \pm 10,4$ & $<0,0001$ & 3,7 \\
\hline Média $(\mathrm{mmHg})$ & $113,7 \pm 12,9$ & $106,1 \pm 11,6$ & $<0,0001$ & 8,8 \\
\hline $\mathrm{FC}(\mathrm{bpm})$ & $76,1 \pm 8,5$ & $74,8 \pm 8,5$ & 0,047 & 2,0 \\
\hline Carga sistólica (\%) & $71,8 \pm 27,9$ & $57,8 \pm 30,3$ & $<0,0001$ & 6,3 \\
\hline Carga diastólica (\%) & $63,2 \pm 28,8$ & $48,4 \pm 30,6$ & $<0,0001$ & 5,4 \\
\hline \multicolumn{5}{|c|}{ Médias durante vigília (7:00 às 23:00h) } \\
\hline Sistólica (mmHg) & $152,2 \pm 17,4$ & $143,7 \pm 15,8$ & $<0,0001$ & 7,4 \\
\hline Diastólica $(\mathrm{mmHg})$ & $96,8 \pm 12,0$ & $90,9 \pm 10,9$ & $<0,0001$ & 7,8 \\
\hline Média (mmHg) & $116,3 \pm 13,2$ & $109,3 \pm 11,8$ & $<0,0001$ & 7,9 \\
\hline $\mathrm{FC}(\mathrm{bpm})$ & $79,2 \pm 8,9$ & $77,3 \pm 8,7$ & 0,009 & 2,7 \\
\hline Carga sistólica (\%) & $69,4 \pm 30,3$ & $55,3 \pm 32,7$ & $<0,0001$ & 5,5 \\
\hline Carga diastólica (\%) & $65,4 \pm 30,7$ & $50,7 \pm 33,6$ & $<0,0001$ & 5,5 \\
\hline \multicolumn{5}{|c|}{ Médias durante sono (23:01 às $6: 59 \mathrm{~h}$ ) } \\
\hline Sistólica (mmHg) & $141,3 \pm 20,4$ & $132,8 \pm 18,1$ & $<0,0001$ & 5,2 \\
\hline Diastólica(mmHg) & $86,2 \pm 12,6$ & $80,6 \pm 11,3$ & $<0,0001$ & 6,9 \\
\hline Média (mmHg) & $105,6 \pm 15,2$ & $99,2 \pm 13,6$ & $<0,0001$ & 4,2 \\
\hline $\mathrm{FC}(\mathrm{bpm})$ & $67,6 \pm 9,0$ & $67,7 \pm 8,3$ & 0,828 & $-0,2$ \\
\hline Carga sistólica (\%) & $79,4 \pm 26,6$ & $65,1 \pm 32,9$ & $<0,0001$ & 4,8 \\
\hline Carga diastólica (\%) & $57,23 \pm 33,29$ & $41,60 \pm 32,26$ & $<0,0001$ & 5,6 \\
\hline
\end{tabular}




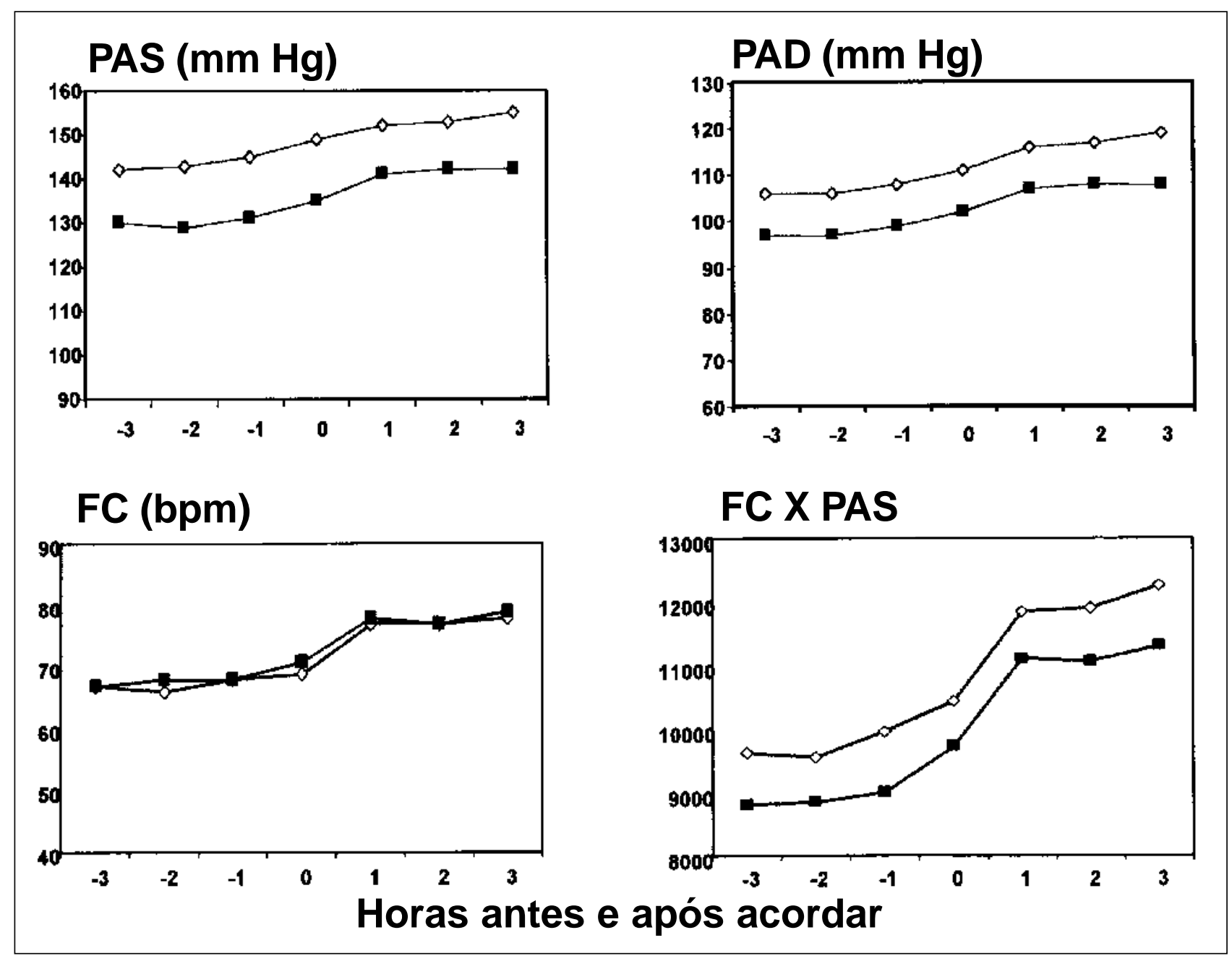

Fig. 2 - Monitorização ambulatorial da pressão arterial (MAPA) após período placebo ( ^) e após tratamento com verapamil COER-24 180/240mg ( $\square$ ) nas 3h antes e após acordar $(\mathrm{n}=81)$. PAS- pressão arterial sistólica; PAD- pressão arterial diastólica; FC- frequiência cardíaca.

\section{Discussão}

Opresente estudo mostrou que verapamilCOER-24 180/ 240mg em dose única ao deitar foi eficaz para a redução dos níveis de pressão arterial, tanto em medidas casuais quanto através da monitorização ambulatorial da pressão arterial de 24h. A tolerabilidade foi considerada boa, uma vez que $68 \%$ dos pacientes não apresentaram eventos adversos.

Verificamos que as maiores reduções da pressão arterial, quando comparamos a monitorização ambulatorial da pressão arterial realizada após o período placebo e a monitorização ambulatorial da pressão arterial após o período de tratamento, ocorreram das 6:00 às 9:00h, ou seja, no período em que os pacientes acordaram, enquanto as menores diferenças ocorreram no horário das 18:00 às 22:00h. Isto é importante, uma vez que já foi demonstrado em estudos anteriores ${ }^{11}$ que o pico de incidência dos eventos mórbidos cardiovasculares ocorre durante a manhã.

Os medicamentos anti-hipertensivos de dose única diária convencionais são preparados para serem administrados pela manhã. Apesar de tais medicamentos manterem nível relativamente prolongado e estável da droga, ocorre, tipicamente, queda gradativa de suas concentrações e de seu efeito até o final do intervalo de $24 \mathrm{~h}$ de administração, que é pela manhã, quando as pressões sistólica e diastólica sobem rapidamente, chegando próximas ao pico do dia ${ }^{12}$. Além disso, a constância pretendida no nível da droga falha quando se leva em consideração a necessidade de variação do controle da pressão arterial, durante o dia versus a noite. Durante o sono, a pressão arterial diminui freqüentemente $15-25 \mathrm{mmHg}$, atingindo em geral, valores normais em pacientes com hipertensão leve e moderada ${ }^{12}$. Uma concentração de medicamento anti-hipertensivo que seja apropriada para o controle da pressão arterial durante o dia pode ser muito grande durante o sono noturno, acarretando o risco de hipotensão noturna com potenciais efeitos indesejáveis.

Recomenda-se que o verapamil COER-24 seja ingerido ao deitar, pois após a sua administração, o revestimento de retenção retarda a liberação do medicamento por aproximadamente $5 \mathrm{~h}$, de maneira que o verapamil seja liberado do comprimido por volta da metade do período de sono do paciente, fazendo com que os níveis mais altos sejam atingidos pela manhã e durante as horas de atividade diurna quando a pressão arterial e o risco de isquemia acham-se mais eleva- 


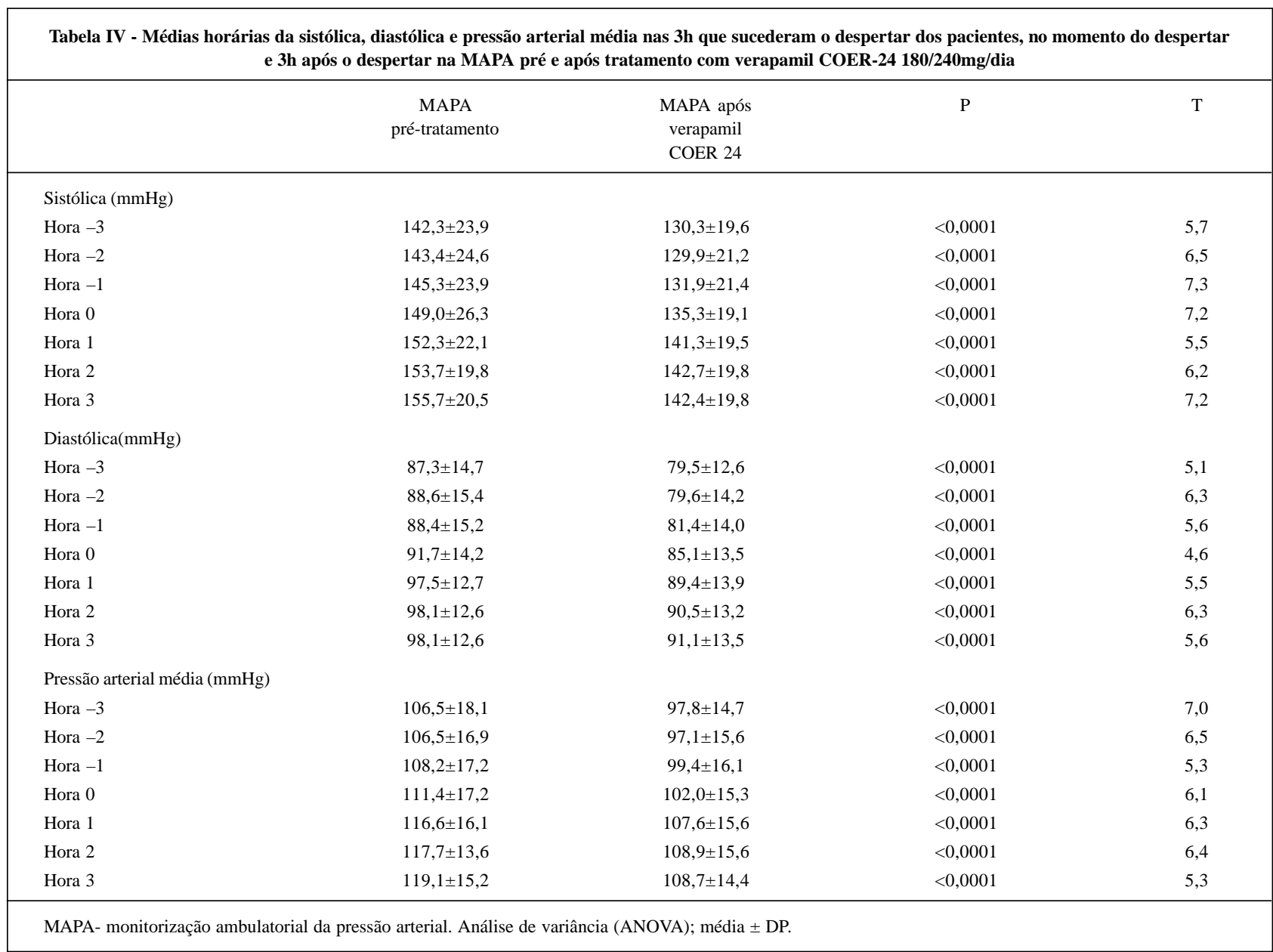

\begin{tabular}{|c|c|c|c|c|}
\hline \multicolumn{5}{|c|}{$\begin{array}{l}\text { Tabela V - Médias horárias da frequiência cardíaca e duplo produto (frequiência cardíaca vs sistólica) nas 3h que suce } \\
\text { o despertar dos pacientes, no momento do despertar e } 3 \mathrm{~h} \text { após o despertar na MAPA pré e após tratamento } \\
\text { com verapamil COER } 24 \text { 180/240 } \mathrm{mg} / \mathrm{dia}\end{array}$} \\
\hline & $\begin{array}{c}\text { MAPA } \\
\text { Pré-tratamento }\end{array}$ & $\begin{array}{c}\text { MAPA } \\
\text { Após verapamil } \\
\text { COER } 24\end{array}$ & $P$ & $\mathrm{t}$ \\
\hline \multicolumn{5}{|c|}{ Frequiência cardíaca (bpm) } \\
\hline Hora -3 & $67,6 \pm 11,7$ & $67,6 \pm 1,0$ & 0,865 & $-0,17$ \\
\hline Hora -2 & $66,8 \pm 11,2$ & $68,1 \pm 10,6$ & 0,911 & $-0,11$ \\
\hline Hora -1 & $68,4 \pm 12,7$ & $68,2 \pm 11,3$ & 0,979 & $-0,03$ \\
\hline Hora 0 & $69,9 \pm 12,5$ & $71,7 \pm 1,4$ & 0,179 & $-1,35$ \\
\hline Hora 1 & $77,9 \pm 14,2$ & $78,2 \pm 17,0$ & 0,879 & 0,15 \\
\hline Hora 2 & $77,4 \pm 13,5$ & $77,3 \pm 2,7$ & 0,305 & $-1,03$ \\
\hline Hora 3 & $78,9 \pm 13,7$ & $79,3 \pm 15,6$ & 0,853 & $-0,19$ \\
\hline \multicolumn{5}{|c|}{ Frequiência cardíaca vs sistólica } \\
\hline Hora -3 & $9634,1 \pm 2398,0$ & $8810,6 \pm 1945,1$ & 0,009 & 2,70 \\
\hline Hora -2 & $9568,4 \pm 2238,7$ & $8852,7 \pm 2029,6$ & 0,011 & 2,60 \\
\hline Hora -1 & $9963,0 \pm 2572,6$ & $9014,0 \pm 2213,6$ & 0,021 & 2,35 \\
\hline Hora 0 & $10438,7 \pm 2794,9$ & $9732,3 \pm 2244,1$ & 0,007 & 2,75 \\
\hline Hora 1 & $11852,5 \pm 2683,1$ & $11110,4 \pm 3139,2$ & $<0,0001$ & 3,96 \\
\hline Hora 2 & $11903,5 \pm 2681,2$ & $11074,6 \pm 2590,0$ & 0,002 & 3,16 \\
\hline Hora 3 & $12262,9 \pm 2639,6$ & $11312,9 \pm 2773,6$ & 0,004 & 3,01 \\
\hline
\end{tabular}


dos. Portanto, a liberação do verapamil deste sistema especial ocorre depois de várias horas; no entanto, a maior parte da dose é eliminada do centro do comprimido dentro de 12 $13 \mathrm{~h}$ após sua ingestão, ao deitar ${ }^{12}$.

De maneira semelhante aos nossos resultados, White e cols. ${ }^{13}$ verificaram em estudo multicêntrico que este novo sistema de liberação do verapamil administrado à noite produziu variações da pressão arterial semelhantes à variabilidade circadiana da pressão arterial: reduções menores mas significativas durante o sono, quando a pressão arterial encontra-se intrinsecamente reduzida na maioria dos hipertensos, e reduções apropriadamente maiores nas horas do despertar pela manhã e durante a vigília. Em outro estudo, White e cols. ${ }^{14}$ encontraram que após o tratamento com verapamil COER 24, a pressão arterial durante o sono diminuiu em maior extensão nos pacientes que não apresentavam, previamente ao tratamento, descenso da pressão arterial durante o sono do que nos pacientes que o apresentavam anteriormente ao tratamento.

Os mesmos autores ${ }^{15}$ verificaram em estudo compara- tivo que tanto o verapamil COER-24 quanto a nifedipina GITS apresentavam redução eficaz da pressão arterial durante $24 \mathrm{~h}$. Porém, o verapamil COER-24 apresentou maiores efeitos do que a nifedipina GITS sobre as variáveis hemodinâmicas no período da manhã (freqüência cardíaca, duploproduto e pressão arterial) e menores efeitos durante o sono, devido às suas propriedades farmacológicas intrínsecas e seu respectivo sistema de liberação.

Portanto, neste estudo a monoterapia com o verapamil COER-24 180/240mg em dose única mostrou-se eficaz para o controle da pressão arterial em hipertensos leves e moderados, demonstrando redução significativa tanto na pressão casual quanto na monitorização ambulatorial da pressão arterial/24h, além de apresentar boa tolerabilidade. Ademais, o verapamil COER-24 180/240mg ao deitar proporcionou reduções maiores e significativas nas primeiras horas da manhã, quando há maior risco de eventos mórbidos cardiovasculares e reduções significativas, porém menores durante o período do sono, demonstrando perfil circadiano favorável.

\section{Referências}

1. Muller JE, Tofler GH, Stone PH. Circadian variation and triggers of onset of acute cardiovascular disease. Circulation 1989; 79: 733-43.

2. Detry JMR, Vincent M. Circadian rhythms in cardiovascular disease: The crucial hours. J Hum Hypertens 1992; 6(suppl 1): S3-S8.

3. Veerman DP, Imholz BPM, Wieling W, Wesseling KH, Van Montfrans GA Circadian profile of systemis hemodynamics. Hypertension 1995; 26: 55-9.

4. White WB. Ambulatory blood pressure monitoring in the evaluation of hypertension therapy. Cardiology Clinics 1995; 13: 569-76.

5. Verdecchia P, Schillaci G, Porcellati C. Dippers versus non-dippers. J Hypertens 1991; 9(suppl 8): S42-S4.

6. Verdecchia P, Schillaci G, Borgioni C, et al. Gender, day-night blood pressure changes, and left ventricular mass in essential hypertension dippers and peakers. Am J Hypertens 1995; 8: 193-6.

7. Bianchi S, Bigazzi R, Baldari G, Sgherri G, Campese VM. Diurnal variations of blood pressure and microalbuminuria in essential hypertension. Am J Hypertens 1994; 7: 23-9.

8. Shimada K, Kawamoto A, Matsubayashi K, Nishinaga M, Kimura S, Ozawa T. Diurnal blood pressure variations and silent cerebrovascular damage in elderly patients with hypertension. J Hypertens 1992; 10: 875-8.

9. Kario K, Matsuo T, Kobayashi H, Imiya M, Matsuo M, Shimada K. Nocturnal fall of blood pressure and silent cerebrovascular damage in elderly hypertensive pa- tients advanced silent cerebrovascular damage in extreme dippers. Hypertension 1996; $27:$ 130-5.

10. Smolensky MH. Chronobiology and chronotherapeutics applications to cardiovascular medicine. Am J Hypertens 1996; 9: 11S-21S

11. Kawano Y, Tochikubo O, Minamisawa K, Miyajima E, Ishii M. Circadian variation of haemodynamics in patients with essential hypertension: comparison between early morning and evening. J Hypertens 1994; 12: 1405-12.

12. Smolensky MH, White WB, Mion Jr. D, Frishman W, Fakouhi TD. Cronobiologia e cronoterapia: novos conceitos na medicina cardiovascular. J Bras Nefrol 1998; 20(suppl. 1): S1-S30.

13. White WB, Anders RJ, Maclntyre JM, Black HR, Sica DA. Verapamil study group. nocturnal dosing of a novel delivery system of verapamil for systemic hypertension. Am J Cardiol 1995; 76: 375-80.

14. White WB, Mehrotra DV, Black HR, Fakouhi TD, COER-verapamil study goup. effects of controlled-onset extended-release verapamil on nocturnal blood pressure (dippers versus nondippers). Am J Cardiol 1997; 80: 469-74.

15. White WB, Black HR, Weber MA, Elliott WJ, Bryzinski B, Fakouhi TD. Comparison of effects of controlled onset extended release verapamil at bedtime and nifedipine gastrointestinal therapeutic system on arising on early morning blood pressure, heart rate, and the heart rate-blood pressure product. Am J Cardiol 1998; 81: 424-31. 